INFLAMMATORY BOWEL DISEASE

\title{
Differential expression of multiple transglutaminases in human colon: impaired keratinocyte transglutaminase expression in ulcerative colitis
}

\author{
G D'Argenio, M Calvani, N Della Valle, V Cosenza, G Di Matteo, P Giorgio, S Margarucci, \\ O Petillo, F P Jori, U Galderisi, G Peluso
}

Gut 2005;54:496-502. doi: 10.1136/gut.2004.049411

See end of article for authors' affiliations

Correspondence to: Professor G Peluso, National Cancer InstituteG, Pascale Foundation, Department of Experimental Oncology, Naples, Italy; g.peluso@ibp.cnr.it

Revised version received 15 July 2004

Accepted for publication 20 July 2004
Background and aims: Ulcerative colitis (UC) is characterised by refractory inflammatory ulceration and damage to the colon. The mechanisms underlying impaired healing have yet to be defined. As transglutaminase expression resulting in matrix protein cross linking is associated with increased wound healing in a rat model of colitis, we hypothesised that different types of transglutaminase might also play a role in UC.

Patients and methods: Endoscopic and histological indices were studied in 26 patients with UC (10 active and 16 inactive) and in 20 normal controls undergoing colonoscopy. Transglutaminase activity was evaluated in plasma (factor XIlla) by a radioenzymatic method. Factor Xllla, tissue and keratinocyte transglutaminase protein content, and mRNA expression in the colon were evaluated by western blot analysis and semiquantitative reverse transcription-polymerase chain reaction (RT-PCR), respectively. Colonic location of transglutaminases and their reaction products, the $\epsilon-(\gamma$-glutamy $))$ lysine bonds, was evaluated by immunohistochemistry using specific monoclonal antibodies.

Results: Transglutaminase activity was significantly lower in the plasma of patients with active UC (4.2 (2.4) $\mathrm{mU} / \mathrm{ml} ; \mathrm{p}<0.05 v$ controls) than in those with inactive UC and controls (10.6 (2.2) and 12.1 (1.7) $\mathrm{mU} / \mathrm{ml}$ ). As shown by western blot, protein levels of tissue transglutaminase and factor XIlla were unchanged in active UC compared with inactive disease and controls, while the keratinocyte form was reduced in active UC. Tissue transglutaminase and factor Xllla immunostaining was strongly present in damaged areas colocalising with isopeptide bonds. In contrast, the keratinocyte form was almost absent in active UC and localised in the upper part of the crypts in normal subjects. RT-PCR showed upregulation of tissue transglutaminase mRNA in active UC (320\% compared with controls) while keratinocyte transglutaminase gene expression was downregulated in active UC.

Conclusions: The results of the present study support the concept that, in the damaged colon, transglutaminases are needed in response to chronic injury and underline the key role of these enzymes in mucosal healing.
A lthough extensive studies have been conducted on the clinical, pathological, and immunological features of IBD, its aetiology and pathogenesis are largely unknown. Transglutaminases, a family of enzymes that catalyse the covalent cross linking between proteins by forming $\epsilon(\gamma$-glutamyl)-lysine isopeptide bonds, have been reported as being actively involved in intestinal mucosal healing. ${ }^{1}$ Indeed, the formation of an isopeptide $\epsilon(\gamma$ glutamyl)-lysine bond between peptide bound glutamine and lysine residues stabilises intra- and extracellular proteins into macromolecular assemblies that are used for a variety of essential physiological purposes such as barrier function in epithelia, apoptosis, extracellular matrix formation, etc. ${ }^{2}{ }^{3}$ The family of transglutaminases comprises several members, including the plasma form (also called coagulation factor XIIIa (FXIIIa)), tissue transglutaminase (tTG), and the keratinocyte membrane bound form. ${ }^{4}$ The plasma transglutaminase molecule, as isolated from blood, is a tetrameric zymogen (FXIII) consisting of two catalytic " $a$ " subunits and two non-catalytic " $b$ " subunits. Thrombin cleavage of the $a$ subunit complex results in the active enzyme composed only of the a subunits. ${ }^{5}$ tTG is a cytosolic enzyme expressed in a variety of tissues and cells and, for its activation, only a critical concentration of calcium is required. ${ }^{5}$
Keratinocyte transglutaminase (TGk), also known as transglutaminase type 1 , is largely membrane bound and was initially described in epidermal keratinocytes. The known biological roles attributed to these enzymes include plasma transglutaminase mediated stabilisation of fibrin gel during blood clotting and TGk cross linking of keratin envelopes formed during epidermal cell differentiation. ${ }^{6}$ tTG has been reported to regulate cell growth and differentiation $^{78}$ and apoptosis. ${ }^{7}$ Transglutaminases also promote the determination of cell shape by cross linking the actin cytoskeleton and its related proteins, ${ }^{9}$ cell adhesion, ${ }^{70}$ and stabilisation of extracellular matrices. ${ }^{11}$ All of the transglutaminases share a large number of substrates, including intracellular proteins such as the actin cytoskeleton ${ }^{12}$ and histones, $^{13}$ and extracellular proteins such as collagen, vitronectin, and fibronectin. ${ }^{14}{ }^{15}$ Many of these are found in the hypertrophic flared matrix of the colonic mucosa, and the extent to which these and other components are cross linked

\footnotetext{
Abbreviations: UC, ulcerative colitis; $\mathrm{TTG}$, transglutaminase; TGk, keratinocyte transglutaminase; FXIII, FXIlla, factor XIII, factor XIlla; CAI, clinical activity index; SDS, sodium dodecyl sulphate; PBS, phosphate buffered saline; RT-PCR, reverse transcription-polymerase chain reaction; HPRT, hypoxanthinephosphoribosyl-transferase
} 
possibly alters the susceptibility of the matrix to proteolysis, and thus affects the rate of its formation and remodelling during mucosal damage and repair. ${ }^{16}$

Both tTG and TGk have been described from well characterised sources in different stratified squamous epithelial and non-epithelial cells and tissues that vary in their properties. ${ }^{17-20}$ Although the presence of tTG has been described in the intestine, ${ }^{21}$ to the best of our knowledge the intestinal localisation of the keratinocyte form has never been described. The functional role of transglutaminases in the human colon is not yet clear but our reports on rats with experimental colitis have documented involvement of tTG and FXIIIa in damaged colonic mucosa, thus possibly affecting mucosal healing capacity. ${ }^{22} 23$

We have extended our earlier experimental data to investigate expression of both tTG and FXIIIa in different phases of ulcerative colitis (UC) concomitantly with levels of isopeptide cross link formed by these enzymes. As rapid resealing of the intestinal barrier after injury is essential for the preservation of well balanced local homeostasis, we also looked at the possible role of TGk in the tight seal between the luminal contents and the underlying mucosal tissues as an important prerequisite of bowel functionality.

\section{METHODS}

\section{Patients}

Twenty six patients with UC diagnosed in the Gastrointestinal Unit of the IRCCS De Bellis (Castellana Grotte, Italy) were included in the study. Median duration of disease was 7.9 years (range six months to 13 years). At the time of recruitment, three patients were not receiving therapy, 23 patients were receiving 5-ASA treatment, and none was receiving concomitant corticosteroids. Fifteen patients had left sided colitis and 11 had extensive colitis (disease extending to the hepatic flexure or beyond). These patients presented no radiological evidence of small bowel disease. Clinical activity index (CAI) was quantified according to modified Rachmilewitz criteria (table 1).

All patients had venesection for routine biochemistry, including determination of $\mathrm{C}$ reactive protein, erythrocyte sedimentation rate, and plasma transglutaminase activity. The disease was considered active if the clinical index was $\geqslant 4$ and was classified as mild (CAI $=4-8)$, moderate (CAI $=9$ $11)$, or severe $(\mathrm{CAI}>11)$. All patients underwent colonoscopy, and endoscopic and histological indices were assessed. At endoscopy (performed by an endoscopist blinded to clinical disease activity) the macroscopic appearance of the mucosa was assessed using an index based on the presence or absence of erythema, oedema, friability, granularity, and erosions, scored on a $0-2$ scale (range $0-10$ ).

Mucosal biopsy specimens were taken from macroscopically affected regions to establish histological disease activity. One pathologist with a special interest in gastrointestinal

\begin{tabular}{lc} 
Table 1 Clinical activity index $(\mathrm{CAI})^{*}$ & \multicolumn{1}{c}{ Range } \\
\hline & $0-2$ \\
\hline Stool frequency/day & $0-2$ \\
Stool consistency & $0-3$ \\
Bloody stools & $0-2$ \\
Stools with mucus & $0-3$ \\
Abdominal pain & $0-2$ \\
Tenesmus & $0-3$ \\
Erythrocyte sedimentation rate & $0-3$ \\
Haemoglobin concentration & $0-2$ \\
White blood cell count & $0-2$ \\
Serum C reactive protein & \\
\hline *CAl: $\leqslant 3=$ inactive; $4-8=$ mild ; $9-11=$ moderate; \\
$>11$ = severe.
\end{tabular}

Table 2 Clinical (CAI), endoscopic, and histological activities of the study group

\begin{tabular}{lc}
\hline Sex (M:F) & $16: 10$ \\
Age (y) of males & $49(27-73)$ \\
Age (y) of females & $46(23-76)$ \\
Duration of the disease (y) & $7.9(6-13)$ \\
No of quiescent patients (CAl =0-3) & 16 \\
No of active patients (CAl $>3)$ & 10 \\
Healthy subjects & 20 \\
Quiescent patients & $1.5(0-3)$ \\
CAl & $0.4(0-1)$ \\
Endoscopic activity & $1.2(0-2)$ \\
Histological activity & $11(9-14)$ \\
Active patients & $4(3-5)$ \\
CAl & $6(5-8)$ \\
Endoscopic activity & \\
Histological activity & \\
\hline &
\end{tabular}

diseases assessed each specimen blind and was kept unaware of the disease activity index of each case. In each specimen, scores on a 0-2 scale were recorded for the severity of accumulation of lymphocytes and plasma cells, infiltration of the crypt epithelium by neutrophils, crypt abscesses, ulceration, mucus depletion of epithelial cells, and crypt distortion (range 0-12). The mean of these scores was obtained for each patient. In all patients, after they had given informed consent, biopsy specimens were also taken for biochemical and immunohistochemical investigation. Clinical, endoscopic, and histological activities of the patients included in the study are summarised in table 2 .

A control group included 20 patients undergoing colonoscopy with a suspected disease that was not confirmed by either endoscopic findings or histological analysis. The control group included patients with other intestinal inflammation such as infectious colitis. The study was approved by the local ethics committee.

\section{Factor XIIIa assay}

Venous blood samples were collected in a citrated tube, and plasma obtained after blood centrifugation was stored at $-20^{\circ} \mathrm{C}$ until assay. According to the method of Muszbek and colleagues, ${ }^{24}$ before the enzyme assay, plasma was treated with bentonite to precipitate fibrinogen and then FXIII was activated to FXIIIa (plasma transglutaminase) by thrombin. Transglutaminase activity in activated plasma was assayed according to a modified version ${ }^{25}$ of the method described by Lorand and colleagues ${ }^{26}: 30 \mu \mathrm{l}$ of sample were added to $45 \mu \mathrm{l}$ of reaction mixture containing a final concentration of $0.25 \mathrm{mM}{ }^{14} \mathrm{C}$-putrescine (Amersham, UK), $50 \mathrm{mM}$ dithiotreitol, $10 \mathrm{mM} \mathrm{CaCl}_{2}$, and $4 \%(\mathrm{w} / \mathrm{v})$ dimethylcasein in Tris $\mathrm{HCl}$ buffer $(50 \mathrm{mM}), \mathrm{pH} 9.0$, with $0.1 \%$ Triton $\times 100$ and incubated with shaking at $37^{\circ} \mathrm{C}$ for 20 minutes. Then, $20 \mu \mathrm{l}$ were spotted onto $3 \mathrm{MM}$ Whatman round paper filters $(2 \mathrm{~cm})$ and immediately plunged into $10 \%$ ice cold trichloroacetic acid for 15 minutes. Two consecutive 15 minute washings were performed in 5\% ice cold trichloroacetic acid followed by a brief washing in ethanol-acetone $(50 \% \mathrm{v} / \mathrm{v})$ and then in acetone. The dried paper filters were counted in $6 \mathrm{ml}$ of Aquasure scintillant (Dupont-NEN). A similar procedure was adopted for blanks, standards, and controls. Transglutaminase units were expressed as $1 \mathrm{mU}=1 \mathrm{nmol}$ of putrescine in acceptor protein at $37^{\circ} \mathrm{C}, \mathrm{pH} 9$.

\section{Antibodies}

The following antibodies were used for identification of tTG, FXIIIa, and TGk: mouse monoclonal anti-tTG antibody (CUB 7402; NeoMarkers, California, USA), rabbit antihuman FXIIIa (generously given by Dr PD Bishop, ZymoGenetics, 
Washington, USA), and antihuman TGk (Biomedical Technologies Inc., Massachusetts, USA). Antibodies were tested for cross reactivity against their corresponding proteins and other members of the transglutaminase family by western blot analysis. None of the three antibodies showed cross reactivity with other forms of transglutaminases. To detect the immunohistochemical localisation of the isopeptide bonds, a mouse monoclonal anti- $\epsilon(\gamma$-glutamyl $)$ lysine isopeptide bond antibody was used (Covalab, Lyon, France).

\section{Western blotting}

Colon biopsy samples were homogenised in RIPA lysis buffer (0.1\% sodium dodecyl sulphate (SDS), $0.5 \%$ deoxycholate, $1 \%$ Nonidet, $100 \mathrm{mM} \mathrm{NaCl}, 10 \mathrm{mM}$ Tris $\mathrm{HCl}$ (pH 7.4)) containing a protease inhibitor cocktail (Sigma, St Louis, Missouri, USA), $0.5 \mathrm{mM}$ dithiotreitol, and $0.5 \%$ phenylmethylsulfonyl fluoride.

Mucosal homogenates from active or inactive UC patients as well as normal controls were run on SDS/polyacrylamide gel according to Laemmli $^{27}$ and transferred to nitrocellulose using Biorad transblot apparatus. tTG, FXIIIa, or TGk primary antibodies were added and incubation continued overnight at $4^{\circ} \mathrm{C}$. The appropriate avidin conjugated secondary antibody was added and immunoreactive proteins were detected by development with the $\mathrm{ABC}$ Vectastain kit (Vector Laboratories, Burlingame, California, USA) according to the manufacturer's instructions.

\section{Immunohistochemistry}

To detect the immunohistochemical localisation of tTG, FXIIIa, and isopeptide bonds, sections from formalin fixed paraffin embedded specimens were deparaffinised and rehydrated in decreasing concentrations of ethyl alcohol. For TGk location, frozen sections were cut $(5 \mu \mathrm{m})$, air dried, and fixed in acetone; slides were rehydrated with phosphate buffered saline (PBS; pH 4). All tissue sections were incubated with fresh $3 \%$ hydrogen peroxide in methanol for 20 minutes and then washed with PBS $(0.1 \mathrm{~mol} / \mathrm{l}$ phosphate and $0.15 \mathrm{~mol} / \mathrm{l}$ sodium chloride, $\mathrm{pH} 7.4)$. Sections were sequentially incubated in $1 \%$ normal blocking serum which was prepared from the species in which the secondary antibody was made, for 30 minutes, and then with specific primary antibody for 60 minutes at room temperature. Subsequent incubations included appropriate biotinylated secondary antibody for 60 minutes and avidin-biotin-peroxidase complex (ABC kit; Vector Laboratories) for 30 minutes followed by the substrate, $3-3^{\prime}$ diaminobenzidine $(10 \mu \mathrm{g} / \mathrm{ml}$ in Tris $\mathrm{HCl}$ buffer, $\mathrm{pH} 7.6$ containing $0.03 \% \mathrm{H}_{2} \mathrm{O}_{2}$ ) for five minutes. Negative controls were obtained by omitting the primary antibody. Sections, counterstained with haematoxylin, were than mounted and observed under light microscopy by a blinded pathologist.

\section{RNA extraction and RT-PCR}

Total RNA was extracted from human biopsy samples using Tri reagent (Molecular Research Center, Inc, Cincinnati, Ohio, USA) according to the manufacturer's protocol. mRNA levels of the genes under analysis were measured by semiquantitative reverse transcription-polymerase chain reaction (RT-PCR) amplification.

Table 3 Transglutaminase (TG) activity in the plasma of patients with ulcerative colitis (UC) and in healthy controls

\begin{tabular}{llll}
\hline & Controls & Inactive UC & Active UC \\
\hline Plasma TG $(\mathrm{mU} / \mathrm{ml})$ & $12.1(1.7)$ & $10.6(2.2)$ & $4.2(2.4)^{*}$ \\
\hline${ }^{*} \mathrm{p}<0.05$. & & & \\
\hline
\end{tabular}

Human mRNA sequences were selected to design primer pairs for RT-PCR reactions using the Oligo 4.05 software (National Biosciences Inc., Plymouth, Minnesota, USA). Primer sequences were as follows: for keratinocyte transglutaminase, upper primer 5' GAC ACA GAC ACA TCC CTT ACC AT 3', lower primer 5' GTC ACT ATT CAC CTC AGC AAA AA $3^{\prime}$ (the size of the amplified fragment was $288 \mathrm{bp}$ ); for tissue transglutaminase, upper primer $5^{\prime}$ CCG TTT TCC ACT AAG AGA TGC 3', lower primer 5' CCC AAA ATT CCA AGG TAT GTT 3' (the size of the amplified fragment was $324 \mathrm{bp}$ ); and for hypoxanthinephosphoribosyl-transferase (HPRT), upper primer 5' CCT GCT GGA TTA CAT CAA AGC ACT G 3', lower primer 5' CCT GAA GTA TTC ATT ATA GTC AAG G 3' (the size of the amplified fragment was $370 \mathrm{bp}$ ).

Amplifications were carried out under the following conditions: $94^{\circ} \mathrm{C}$ for 45 seconds, $57^{\circ} \mathrm{C}$ for 45 seconds, and $72^{\circ} \mathrm{C}$ for 45 seconds. The number of cycles varied according to the expression level of the target gene. The correct number of cycles was determined previously to ensure that PCR was taking place in the linear range in order to guarantee a proportional relationship between input RNA and densitometric readout. Each RT-PCR experiment was repeated at least three times. Amplification products were electrophoresed on $2 \%$ agarose gel in $1 \times$ TAE. Semiquantitative analysis of mRNA levels was carried out using the software associated with the Fluor S imager (BioRad Company, Hercules, California, USA). The density values of RT-PCR products were normalised with respect to endogenous HPRT product levels.
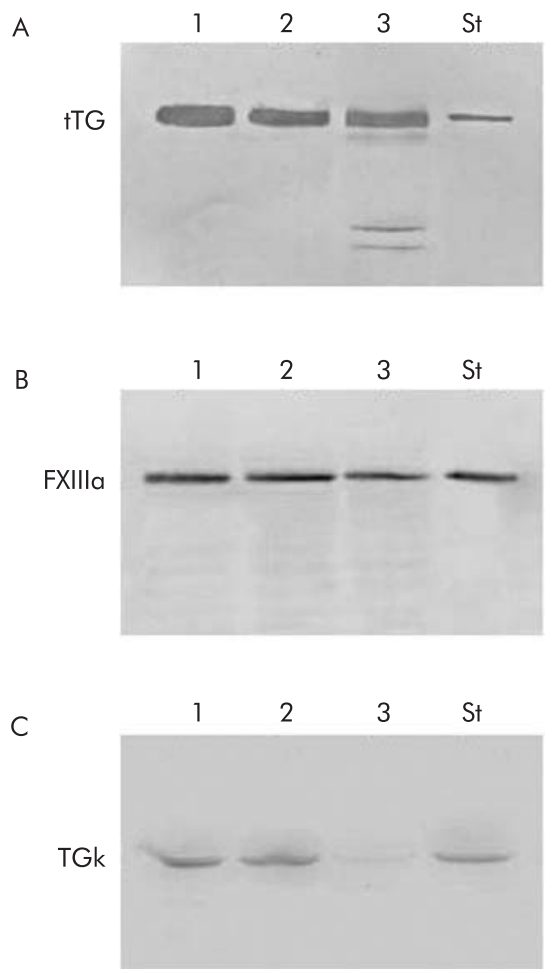

Figure 1 Western blot analysis using antibodies against tissue transglutaminase ( $\mathrm{tTG}$ ( $(\mathrm{A})$, factor XIlla (FXIlla) (B), and keratinocyte transglutaminase (TGK) (C). Lane 1: control subjects; lane 2: inactive ulcerative colitis; lane 3: active ulcerative colitis; St, standard. In active ulcerative colitis, the protein contents of TTG and FXIlla were almost unchanged or slightly reduced compared with controls. TGk expression was strongly reduced compared with inactive colitis and control subjects. 
Purification of insoluble high molecular weight proteins and measurement of isopeptide cross link Insoluble proteins obtained from frozen tissue sections boiled for 10 minutes in an extraction solution consisting of $2 \%$ $(\mathrm{w} / \mathrm{v})$ SDS and $0.1 \%(\mathrm{w} / \mathrm{v})$ dithiotreitol were sedimented by centrifugation for five minutes at $13500 \mathrm{~g}$. The pellet was then resuspended and extracted three more times and finally suspended in $0.1 \mathrm{M}$ N-ethylmorpholine acetate $(\mathrm{pH} 8.3)$. An aliquot $(10 \%)$ was used for quantitation of total protein amount by amino acid analysis while another $30 \%$ was subjected to total enzymatic digestion to release the free $\epsilon(\gamma$ glutamyl)-lysine isopeptide cross link. The isopeptide was then resolved and quantitated by amino acid analysis, ${ }^{28}$ considering that it elutes immediately after methionine. In a related set of experiments, the isopeptide content of tissue samples was determined without prior extraction.

\section{RESULTS}

\section{Factor XIIla activity}

To determine whether plasma transglutaminase enzymatic activity is changed during the active inflammatory phase of UC, we used the standard assay measuring ${ }^{14} \mathrm{C}$-putrescine incorporation into casein. Transglutaminase activity was strongly reduced in the plasma of patients with active UC and returned to normal values in inactive patients (table 3). There was no statistically significant difference in FXIIIa activity in patients with extensive colonic disease compared with patients with distal disease (not shown). This result is in keeping with similar findings previously reported by others and by ourselves ${ }^{29}{ }^{30}$ and suggest that in the damaged colonic

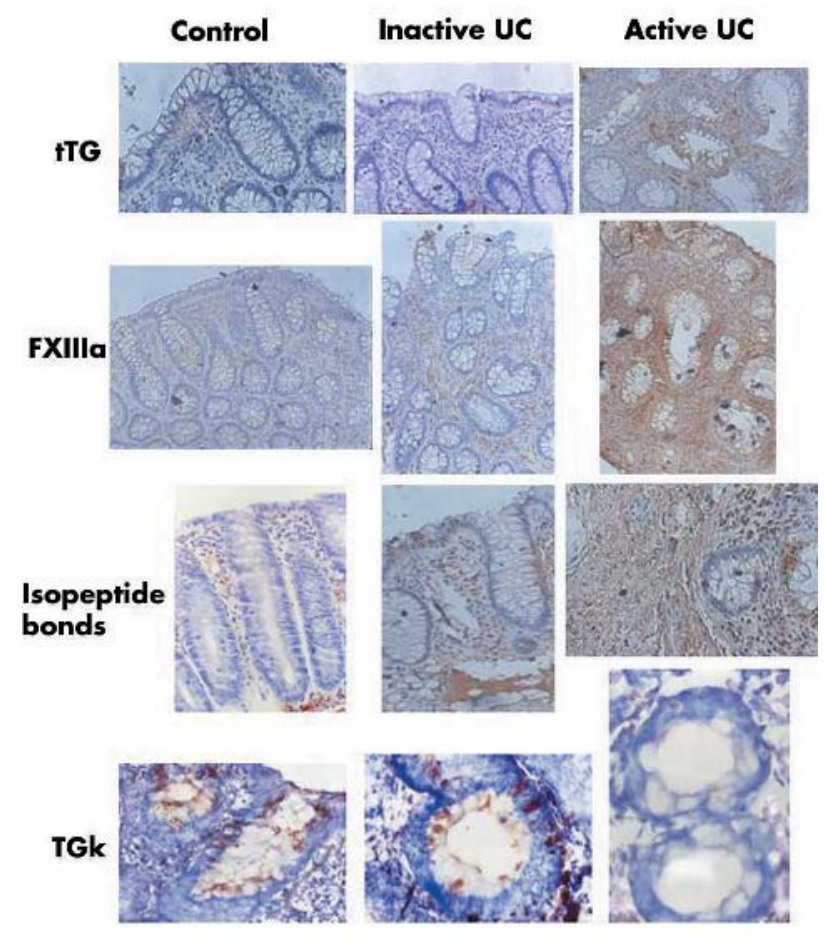

Figure 2 Immunohistochemistry showing the distribution of different forms of transglutaminases and isopeptide bonds (as their products of reaction) in active and inactive ulcerative colitis (UC) and in control subjects. Tissue transglutaminase (tTG), factor XIIla (FXIIla), and isopeptide bonds were markedly increased in active UC and widely distributed in the extracellular matrix (original magnification 200×). In contrast, the keratinocyte isoform (keratinocyte transglutaminase (TGk)) was almost absent in active UC and localised in the upper crypt colonocytes of control subjects (original magnification $400 \times$ ). mucosa a large amount of plasma transglutaminase (and presumably also the tissue form) is released into the matrix.

\section{Protein expression}

To address the question of whether different forms of transglutaminases are present in damaged colonic mucosa and whether tissue inflammation also influences their expressions, protein levels of tTG, TGk, and plasma FXIIIa were assayed by western blotting. Western blot analysis revealed for the first time the presence of three isoforms of transglutaminase in the human colon. Compared with control subjects, tTG protein levels in active UC appeared with two bands of degradation, probably due to endogenous proteolysis by calpain which is known to be increased in active colitis. ${ }^{31}$ tTG expression returned towards normal levels in inactive UC patients. FXIIIa protein levels were substantially unchanged in active and inactive UC compared with control subjects. Moreover TGk expression was reduced in active UC but returned towards normal levels in inactive UC patients. These results were reproduced for all patients involved in the study in independent experiments and a representative western blot for each isoform of transglutaminase is shown in fig $1(\mathrm{~A}-\mathrm{C})$.

\section{Immunohistochemistry}

To assess the distribution of different forms of transglutaminases in the colons of patients with UC and in normal subjects, we performed immunohistochemical analyses using TTG, FXIIIa, and TGk antibodies. We also examined the distribution of the $\epsilon(\gamma$-glutamyl) lysine isopeptide bond as the final reaction product catalysed by transglutaminases using an anti-isopeptide antibody (fig 2). In control subjects, tTG was only slightly expressed in colonocytes and in the basal membrane of the epithelial surface while FXIIIa was almost absent except for staining a few inflammatory cells; staining of isopeptide bonds was confined to intercellular spaces and to the basal membrane of the epithelium. In patients with active UC, strong positive staining was found for both tTG and FXIIIa in the extracellular matrix and perivascular stroma, mainly surrounding mucosal lesions. Damaged or distorted crypt colonocytes also stained positive for tTG. Extracellular localisation of tTG and FXIIIa was almost the same with a prevalence of FXIIIa on perivascular areas. The immunohistochemical pattern of the $\epsilon(\gamma$-glutamyl) lysine isopeptide bond in active UC coincided with the extracellular distribution of FXIIIa and tTG. Staining for tTG,

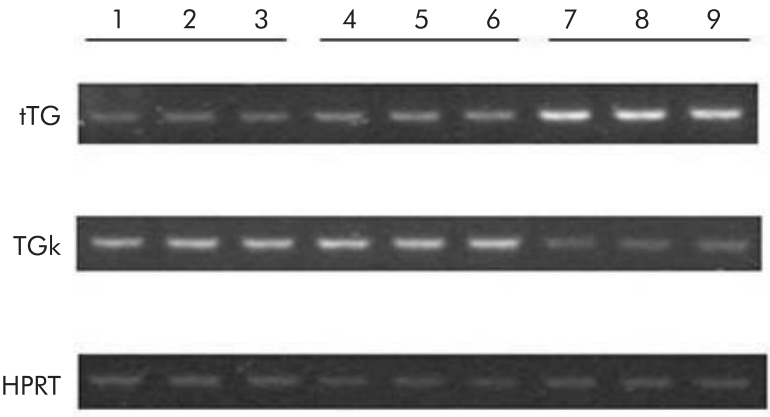

Figure 3 Agarose gel electrophoresis of representative reverse transcription-polymerase chain reaction products of tissue transglutaminase (tTG), keratinocyte transglutaminase (TGk), and hypoxanthinephosphoribosyl-transferase (HPRT). HPRT was chosen as an internal control. Lanes 1-3: control subjects; lanes 4-6: inactive ulcerative colitis; lanes 7-9: active ulcerative colitis. TTG mRNA was upregulated in active ulcerative colitis compared with control subjects while TGk showed the opposite expression pattern. 
FXIIIa, and isopeptide bonds in inactive colitis was similar to that of control subjects.

Finally, we showed for the first time the presence of TGk in the colonic mucosa. In control subjects, an increasing number of epithelial cells positive for TGk were observed from the base to the upper crypt; staining was less pronounced in inactive patients and surprisingly absent in active UC. The extracellular matrix was invariably negative for TGk.

\section{RNA extraction and RT-PCR}

We determined mRNA levels of tTG and TGk by semiquantitative RT-PCR to ascertain whether expression of these genes was modified in the colonic mucosa of patients with UC. We found that TTG mRNA was significantly more abundant in patients with UC, with an increase ranging from 2 to 4 times compared with control subjects. mRNA levels of tTG in inactive patients were intermediate between active UC and control subjects.

TGk displayed the opposite expression pattern. mRNA levels slightly decreased in the colonic mucosa of inactive patients and showed a significant reduction in active UC with respect to controls. A representative RT-PCR amplification pattern for each isoform of transglutaminase is shown in fig 3 .

\section{Isolation of high molecular weight proteins from UC tissue containing isopeptide cross links}

We investigated whether increased expression of transglutaminases in UC contributed to the formation of cross linked protein deposits. To confirm that the high molecular weight proteins arise from transglutaminase cross linking, we employed the method established for isolation of the insoluble cornified cell envelope of the epidermis. Colon biopsies were exhaustively extracted by boiling with a solution containing $2.0 \%$ SDS and $0.1 \%$ dithiotreitol, and insoluble proteins were collected by centrifugation. At amino acid analysis, the insoluble proteins constituted 15\% of the total protein of the UC colon tissue sections. In contrast, we could recover only trace amounts of insoluble proteins from normal tissue samples ( $0.1 \%$ of total protein). An aliquot of insoluble proteins from UC tissues was then subjected to total enzymatic digestion to release the free isopeptide cross link. Following quantitation by amino acid analysis, we found 1.2 residues of cross link/100 residues, very similar to the 1 residue/100 residues detected in the skin. Furthermore, the total amounts of cross link in intact tissue sections were 1-2 and 10-11 residues of cross link/10 000 residues in normal and UC tissues, respectively. The former value, which is similar to that found in other normal tissues such as the liver, may represent the background value. The latter value is consistent with the content of insoluble proteins recovered from UC tissues and thus indicates that the higher levels of cross link in UC tissue originate from insoluble proteins. Taken together, the results of the present study show that at least three members of the transglutaminase enzyme family are present in colon tissue and are actively involved in the remodelling /healing process, as we suggest in fig 4 .

\section{DISCUSSION}

In this study, we have shown that three forms of transglutaminases are present in the human colon and modify their expression and tissue distribution in damaged mucosa of patients with UC, being involved in mucosal remodelling/ healing and colonocyte adaptive processes. We have demonstrated the presence of large amounts of tTG and plasma FXIIIa in the extracellular matrix of damaged tissue, concomitant with significant levels of isopeptide cross link formed by these enzymes. We have also demonstrated for the first time the presence of the keratinocyte form of transglutaminase in human normal colonocytes whereas it was not expressed in active colitis.

Transglutaminases form a family of enzymes that have evolved for specialised functions such as protein cross linking in haemostasis, wound healing, apoptosis, or keratinocyte cornified envelope formation. Cross linking activity is involved in disparate biological phenomena depending on

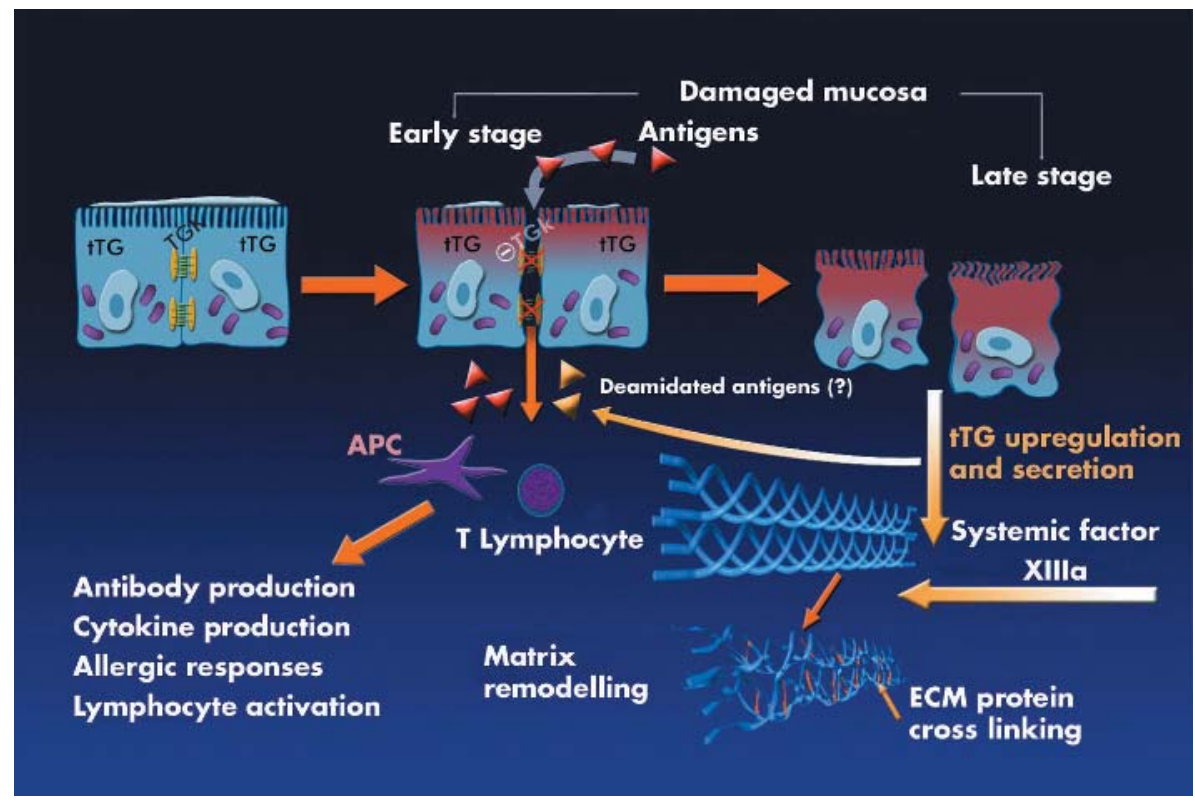

Figure 4 Proposed role of different transglutaminases in normal or damaged colonic mucosa. In the normal colonic mucosa, keratinocyte transglutaminase (TGk) contributes to intercellular tight junction formation while tissue transglutaminase ( $\mathrm{tTG}$ ) is preferentially localised within the cell cytosol. In the presence of pathogens, TGk is downregulated allowing cell separation and antigens to penetrate and perpetrate the mucosal damage. At that stage, $\mathrm{TTG}$ is upregulated and massively released in the inflamed area together with the circulating transglutaminase factor XIlla; they promote cross linking of proteins in the extracellular matrix (ECM) initiating the healing process. 
the location of the target proteins. Intracellular activation of tTG and TGk can give rise to cross linked protein envelopes in apoptotic and cornified epidermal cells, respectively. Moreover, extracellular activation of tTG or plasma FXIIIa contributes to the stabilisation of the extracellular matrix and promotes cell-substrate interaction and wound healing.

While the presence of tTG and plasma FXIIIa is well established in colon tissue, the present study is the first report on the involvement of TGk. TGk is abundantly expressed in terminally differentiating stratified squamous epithelia where it is required for barrier function by formation of an envelope by cross linking a series of defined structural proteins. ${ }^{31-34}$ Almost all of the enzyme is membrane bound.$^{35-37}$ During differentiation, some of it is proteolytically processed into forms with up to a 200 -fold increase in specific activity. ${ }^{37}$ The properties of tTG are similarly complex. This protein serves as the $G$ protein involved in signal transduction in most mammalian cells. ${ }^{38}$ However, when $\mathrm{Ca}^{2+}$ concentrations rise significantly above normal intracellular levels, this ubiquitous protein becomes active in cross linking transglutaminase reactions ${ }^{32} 33$ and is largely cytosolic. ${ }^{2-33}$ Furthermore, it cannot be activated by any known process and, unlike TGk or plasma FXIIIa, it is inactivated when proteolysed by a specific protease (that is, calpain). ${ }^{39}$ Indeed, tTG is an in situ substrate of calpain ${ }^{39}$ and the finding that treatment of experimental colitis with calpain inhibitor I reduces colon injury ${ }^{31}$ suggests that the increased availability of active tTG at the site of damage is a pathway through which the repairing process take place. Based on the known properties of these enzymes, our new data suggest that most of the transglutaminases present in colon tissue from UC are related to tTG and plasma FXIIIa as TGk is significantly downregulated at the transcriptional level and almost absent by western blot analysis and immunohistochemical study. Two types of data reported here point to the conclusion that this abnormal transglutaminase expression (decrease in TGk and increase in tTG) could be associated with the course of UC.

Firstly, immunohistochemisty showed striking colocalisation of large deposits of cross linked material (positive for $\epsilon$ ( $\gamma$-glutamyl)-lysine isopeptide) and tTG and plasma FXIIIa deposition. Indeed, our study showed that the ultimate fate of tTG and plasma FXIIIa in active UC was to be released into the matrix. There, most of the transglutaminase cross links are in the extracellular matrix with fibronectin, ${ }^{40}{ }^{41}$ collagen, laminin, fibrin, and many other substrates. Indeed, a recent elegant study demonstrated that tTG binds to pericellular fibronectin through its amino terminal $\beta$-sandwich domain. ${ }^{42}$ Accordingly, our immunohistochemical analysis using antibody against the major cross link produced by the enzyme (the $\gamma$-Glu- $\epsilon$-Lys dipeptide) showed increased isopeptide bonds that colocalised with tTG and plasma FXIIIa in the extracellular matrix of damaged tissue. This was in agreement with upregulation of tTG mRNA and depletion of plasma FXIIIa levels in blood while we found tTG protein degradation by western blot analysis in inflamed colonic mucosa. Taken as a whole, these data support the role of tTG and plasma FXIIIa in intestinal mucosa healing of UC patients. Wound healing is based on extracellular matrix remodelling that consists of a destructive part, mainly catalysed by extracellular proteinases, such as MMPs and calpain, and of a constructive part that involves processing enzymes such as transglutaminases. The extent to which the components of the extracellular matrix are cross linked alters the susceptibility of the matrix to proteolysis and thus affects the rate of its formation and remodelling during mucosal damage; fibronectin, for example, can be cleaved by MMPs only after it has been cross linked by transglutaminase. ${ }^{16}$
Thus increased transglutaminase availability is useful in the formation of "highly remodelling" matrices under conditions of continual wound healing such as in UC. On the other hand, over expression of tTG could be failing due to the presence of proteinases in inflamed mucosa that, besides proteolysis of extracellular matrix protein, inactivate at least part of the product of tTG gene expression, as demonstrated by western blot analysis. Indeed, enzyme degradation and inactivation may account for the apparent discrepancy between tTG gene upregulation observed in UC and decreased TG activity reported in our earlier studies in colonic tissue in experimental colitis in rats. ${ }^{22}{ }^{23}$ This hypothesis is strongly supported by our findings that treatments with Na-butyrate, a short chain fatty acid that upregulates tTG gene expression, and recombinant FXIIIa increase transglutaminase activity in intestinal mucosa and ameliorate colitis both in humans and in rat models. ${ }^{1} 234344$ In this regard, in the intestine, both FXIII and tTG have been reported to contribute to the extracellular matrix assembly in active Crohn's disease. ${ }^{45}$ Indeed, the complex interactions between extracellular matrix components, including constitutive proteins and enzymes and growth factors, should be better addressed in order to understand the mechanism of the healing processes affecting the course of UC.

Secondly, we demonstrated a specific role for TGk, which is missing in active UC. To the best of our knowledge, expression of TGk in human tissue is limited essentially to stratified squamous epithelia where it appears in substantial amounts, typically in cells midway between the basal layer and the callus layer. ${ }^{18}{ }^{46}$ Keratinocytes are the principal type of cell in stratified squamous epithelia such as the epidermis. The terminally differentiated keratinocytes of the outermost (callus) layer of the epidermis, constituting the main extrapulmonary protective barrier against the environment, consist largely of disulphide bonded hydrophobic keratin intermediate filament proteins. Surrounded by envelopes consisting of cross linked protein, these cells are remarkably cohesive ${ }^{47}$ a property which is even more obvious in hair and nail. In this study, for the first time we reported the presence of TGk in the upper part of the crypt in the normal colon, suggesting that in a manner similar to the role played in the epidermis, TGk may promote cell cohesion in the colon epithelium. On the other hand, even if the characteristics of colonic epithelium are different from those of the epidermis, they share a similar barrier function that may explain the presence of TGk in both tissues. In active colitis, TGk is neither expressed in colonocytes nor released in the extracellular matrix, as occurs with tTG. Although the role of TGk in the colon should be better defined in relation to its potential substrates, our finding that damaged epithelium with increased permeability to bacterial and luminal antigenic components shows impaired TGk expression as a marker of reduced cell adhesion remains of considerable interest.

Epithelial cells express six of the nine members of the transglutaminase family, and this study showed that at least three are important in intestinal biology. The present study supports the concept that transglutaminases are needed in response to chronic injury in the damaged colon and underline the key role of these enzymes in mucosal remodelling/healing and colonocyte adaptive processes. We postulate that therapies affecting enzyme activity in the colonic mucosa may contribute to improving the repair response in UC.

\section{ACKNOWLEDGEMENTS}

This paper was partly supported by a grant from the "Ministero della Salute: Progetto finalizzato n.31" 


\section{Authors' affiliations}

G D'Argenio, N Della Valle, V Cosenza, Gastroenterology Unit, School of Medicine, Federico II University, Naples, Italy

M Calvani, Scientific Department Sigma-Tau, Pomezia, Roma, Italy G D Matteo, P Giorgio, Digestive Endoscopy Unit, IRCCS S De Bellis, Castellana Grotte, Italy

S Margarucci, IBP CNR, Naples, Italy

O Petillo, IBP CNR, Naples, Italy, and National Cancer Institute-G, Pascale Foundation, Department of Experimental Oncology, Naples, Italy

F P Jori, G Peluso, National Cancer Institute-G Pascale Foundation, Department of Experimental Oncology, Naples, Italy

U Galderisi, 2nd University of Naples, Department of Experimental Medicine, Naples, Italy

Conflict of interest: None declared.

\section{REFERENCES}

1 D'Argenio G, Cosenza V, Sorrentini I, et al. Butyrate, mesalamine, and factor XIII in experimental colitis in the rat: effects on transglutaminase activity. Gastroenterology 1994:106:399-404.

2 Folk JE. Transglutaminases. Annu Rev Biochem 1980;49:517-31.

3 Aeschlimann D, Paulsson M. Cross-linking of laminin-nidogen complexes by tissue transglutaminase. A novel mechanism for basement membrane stabilization. J Biol Chem 1991;266:15308-17.

4 Lorand L, Graham RM. Transglutaminases: crosslinking enzymes with pleiotropic functions. Nature 2003;4:140-56.

5 Fesus L, Madi A, Balajthy Z, et al. Transglutaminase induction by various cell death and apoptosis pathways. Experientia (Basel) 1996;52:942-9.

6 Thacher SM. Purification of keratinocyte transglutaminase and its expression during squamous differentiation. J Invest Dermatol 1989;92:578-84

7 Borge L, Demignot S, Adolphe M. Type II transglutaminase expression in rabbit articular chondrocytes in culture: relation with cell differentiation, cell growth, cell adhesion and cell apoptosis. Biochim Biophis Acta 1996:1312:117-24.

8 Katoh S, Nakagawa N, Yano Y, et al. Hepatocyte growth factor induces transglutaminase activity that negatively regulates the growth signal in primary cultured hepatocytes. Exp Cell Res 1996;222:255-61.

9 Nemes Z Jr, Adany R, Balazs M, et al. Identification of cytoplasmic actin as an abundant glutaminyl substrate for tissue transglutaminase in $\mathrm{HL}-60$ and $\mathrm{U} 937$ cells undergoing apoptosis. J Biol Chem 1997;272:20577-83.

10 Ueki S, Takagi J, Saito Y. Dual functions of transglutaminase in novel cell adhesion. J Cell Sci 1996;109:2727-35.

11 Aeschlimann D, Kaupp O, Paulsson M. Transglutaminase-catalyzed matrix cross-linking in differentiating cartilage: identification of osteonectin as a major glutaminyl substrate. J Cell Biol 1995;129:881-92.

12 Zhu Y, Tassi L, Lane W, et al. Specific binding of the transglutaminase, platelet factor XIII, to HSP72. J Biol Chem 1994;269:22379-84.

13 Ballestar $E$, Abad C, Franco L. Core histones are glutaminyl substrates for tissue transglutaminase. J Biol Chem 1996;271:18817-24.

14 Mosher DF, Proctor RA. Binding and factor XIlla-mediated cross-linking of a 27-kilodalton fragment of fibronectin to Staphylococcus aureus. Science 1980;209:927-9.

15 Aeschlimann D, Mosher D, Paulsson M. Tissue transglutaminase and factor $\mathrm{XIII}$ in cartilage and bone remodeling. Semin Thromb Hemost 1996;22:437-43

16 Bini A, Itoh Y, Kudryk BJ, et al. Degradation of cross-linked fibrin by matrix metalloproteinase 3 (stromelysin 1): hydrolysis of the gamma Gly 404-Ala 405 peptide bond. Biochemistry 1996;35:13056-63

17 Chang SK, Chung SI. Cellular transglutaminase. The particulate-associated transglutaminase from chondrosarcoma and liver: partial purification and characterization, J Biol Chem 1986;261:8112-21.

18 Parenteau NL, Pilato A, Rice RH. Induction of keratinocyte type-I transglutaminase in epithelial cells of the rat. Differentiation 1986;33:130-41.

19 Schmidt R, Michel R, Shroot B, et al. Transglutaminases in normal and transformed human keratinocytes in culture. J Invest Dermatol 1988;90:475-9.

20 Cardinali M, Uchino R, Chung SI. Interaction of fibrinogen with murine melanoma cells: covalent association with cell membranes and protection against recognition by lymphokine-activated killer cells. Cancer Res 1990;24:8010-16.

21 D'Argenio G, Sorrentini I, Ciacci C, et al. Transglutaminase activity along the rat small bowel and cellular location. Enzyme 1988;39:227-30.

22 D'Argenio G, Sorrentini I, Cosenza V, et al. Serum and tissue transglutaminase correlates with the severity of inflammation in induced colitis in the rat. Scand J Gastroenterol 1992;27:111-14.

23 D'Argenio G, Grossman A, Cosenza V, et al. Recombinant factor XIII improves experimental colitis in rats. Dig Dis Sci 2000:45:987-97.

24 Muszbek L, Polgar J, Fesus L. Kinetic determination of blood coagulation factor XIII in plasma. Clin Chem 1985;31:35-40.

25 Bruce SE, Bjarnason I, Peters TJ. Human jejunal transglutaminase: demonstration of activity, enzyme kinetics and substrate specificity with special relation to gliadin and celiac disease. Clin Sci 1985;68:573-9.

26 Lorand L, Campbell-Wilkes LK, Cooperstein L. A filter paper assay for transamidating enzymes using radioactive amine substrates. Ann Biochem 1972;50:623-31

27 Laemmli UK. Cleavage of structural proteins during the assembly of the head of bacteriophage T4. Nature 1970;227:680-5.

28 Hohl D, Mehrel T, Lichti U, et al. Characterization of human loricrin. Structure and function of a new class of epidermal cell envelope proteins. J Biol Chem $1991 ; 266: 6626-36$

29 Standnicki A, Kloczko J, Nowak A, et al. Factor XIII subunits in relation to some other hemostatic parameters in ulcerative colitis. Am J Gastroenterol $1991 ; 86: 690-3$.

30 D'Argenio G, Ciacci C, Sorrentini I, et al. Serum transglutaminase in inflammatory bowel disease. J Clin Gastroenterol 1990;12:400-4

31 Cuzzocrea S, Mc Donald MC, Mazzon E, et al. Calpain inhibitor I reduces colon injury caused by dinitrobenzene sulphonic acid in the rat. Gut 2001;48:478-88.

32 Aeschlimann D, Mosher D, Paulsson M. Tissue transglutaminase and factor XIII in cartilage and bone remodeling. Semin Thromb Hemostasis 1996;22:437-43

33 Melino G, Candi E, Steinert PM. Assay for transglutaminases in cell death. Methods Enzymol 2000;322:433-72.

34 Rice RH, Green H. Presence in human epidermal cells of a soluble protein precursor of the cross-linked envelope: activation of the cross-linking by calcium ions. Cell 1977;11:417-22.

35 Kim SY, Chung SI, Steinert PM. Highly active soluble processed forms of the transglutaminase 1 enzyme in epidermal keratinocytes. J Biol Chem 1995;270: 18026-35.

36 Steinert PM, Kim SY, Chung SI, et al. The transglutaminase 1 enzyme is variably acylated by myristate and palmitate during differentiation in epidermal keratinocytes. J Biol Chem 1996;271:26242-50.

37 Steinert PM, Chung SI, Kim SY. Inactive zymogen and highly active proteolytically processed membrane-bound forms of the transglutaminase 1 enzyme in human epidermal keratinocytes. Biochem Biophys Res Commun 1996;221:101-6.

38 Nakaoka H, Perez DM, Baek KJ, et al. Gh: a GTP-binding protein with transglutaminase activity and receptor signaling function. Science 1994;264:1593-6.

39 Zhang J, Guttmann RP, Jhonson GVW. Tissue transglutaminase is in situ substarte of calpain: regulation of activity. J Neurochem 1998;71:240-7.

40 Beninati S, Senger DR, Cordella-Miele E, et al. Osteopontin: its transglutaminase-catalyzed posttranslational modifications and crosslinking to fibronectin. J Biochem (Tokyo) 1996;115:675-82.

41 Komarek D, Achyuthan TC, Rowland TC, et al. Hierarchies in the binding of human factor XIII, factor XIIla, and endothelial cell transglutaminase to human plasma fibrinogen, fibrin, and fibronectin. Mol Cell Biochem 1996;162:43-9.

42 Gaudry CA, Verderio E, Aeschlimann D, et al. Cell surface localization of tissue transglutaminase is dependent on a fibronectin-binding site in its $\mathrm{N}$ terminal beta-sandwich domain. J Biol Chem 1999:274:30707-14.

43 Scheppach W, Sommer H, Kirchner T, et al. Effect of butyrate enemas on the colonic mucosa in distal ulcerative colitis. Gastroenterology 1992;103:51-6.

44 Lorenz R, Heinmuller $M$, Classen $M$, et al. Substitution of factor XIII: a therapeutic approach to ulcerative colitis. Haemostasis 1991;21:5-9.

45 D'Argenio G, Biancone L, Cosenza V, et al. Transglutaminases in Crohn's disease. Gut 1995;37:690-5.

46 Thacher SM, Rice RH. Keratinocyte-specific transglutaminase of cultured human epidermal cells: relation to cross-linked envelope formation and terminal differentiation. Cell 1985:40:685-95.

47 Rothnagel JA, Rogers GE. Transglutaminase-mediated cross-linking in mammalian epidermis. Mol Cell Biochem 1984;58:113-19. 